\title{
Electromagnetically Induced Guiding of Counter-Propagating Lasers in Plasmas
}

\author{
G. Shvets \\ Princeton Plasma Physics Laboratory, Princeton University, Princeton, NJ 08543
}

A. Pukhov

Max-Plank-Institut fur for Quantenoptik, D-85\%48 Garching, Germany

\begin{abstract}
The interaction of counter-propagating laser pulses in a plasma is considered. When the frequencies of the two lasers are close, nonlinear modification of the refraction index results in the mutual focusing of the two beams. A short (of order the plasma period) laser pulse can also be nonlinearly focused by a long counter-propagating beam which extends over the entire guiding length. This phenomenon of electromagnetically-induced guiding can be utilized in laser-driven plasma accelerators.
\end{abstract}

Submitted for publication, 1998. 
The past decade witnessed a dramatic increase in intensities of laboratory-scale laser systems $[1,2]$, stimulating significant interest in the nonlinear optics of plasmas [3-5]. One of the areas of active research is propagation of intense laser pulses over long distances in plasmas because of its importance for a number of applications, including laser wakefield particle accelerators [6]. A laser will, in free space, remain focused over a diffraction length (Rayleigh range) $Z_{r}=\pi \sigma^{2} / \lambda_{0}$, where $\lambda_{0}$ is the laser wavelength, and $\sigma$ is the laser spot size at the focus. Nonlinear effects, such as relativistic and ponderomotive self-focusing, which can overcome diffraction, have been studied theoretically [7,8] and observed experimentally [9]. Nonlinear self-focusing is ineffective for short laser pulses [10] and requires a very high laser power $P_{c 1}=17 \omega_{0}^{2} / \omega_{p}^{2} \mathrm{GW}$, where $\omega_{p}^{2}=4 \pi n_{0} e^{2} / m$ is the plasma frequency, $n_{0}$ is the background electron density, and $-e$ and $m$ are the electron density and mass, respectively.

In this Letter we demonstrate how a laser pulse can be guided through the plasma without diffraction due to its nonlinear interaction with another, counter-propagating pulse. Such electromagnetically induced guiding (EIG) occurs at laser intensities much below the relativistic threshold $P_{c 1}$. Nonlinear interaction of the guiding and guided beams generates a plasma density grating which backscatters the guiding pulse. The interference of this backscattered wave with the guided pulse results in the channeling of the latter. Two distinct problems are analyzed in this Letter: (i) mutual guiding of two long counterpropagating laser pulses and (ii) guiding of an ultra-short tightly focused laser pulse by a counter-propagating, lower intensity Bessel beam. In both cases the length of the long (guiding) beam is approximately twice the desired propagation distance.

The technique of employing a second laser pulse to change the propagation properties of the first pulse is widely known in conventional (atomic) nonlinear optics. For example, by utilizing the effect of electromagnetically induced transparency (EIT) [14], the medium which is opaque to the laser pulse taken alone can become transparent in the presence of the second pulse. As was recently demonstrated by Harris [4], a similar process can take place in cold electron plasmas, where the two lasers have to be detuned by $\Delta \omega \approx \omega_{p}$. The transverse profile of the nonlinear index of refraction can be changed by the second pulse 
to prevent, e. g., an intense pulse from self-filamenting [15]. EIG of co-propagating lasers in plasma was also considered by Gibbon and Bell [16]; the required laser power in that case is, however, much larger than in the counter-propagating case, and guiding of pulses shorter than a plasma wavelength is impossible.

Consider two circularly polarized counter-propagating laser pulses $\vec{a}_{0}$ and $\vec{a}_{1}$, where $\vec{a}_{0,1}=a_{0,1} / 2\left(\vec{e}_{x} \pm i \vec{e}_{y}\right) e^{i \theta_{0,1}}+$ c.c. are the normalized vector potentials: $a_{0,1}\left(\vec{r}_{\perp}, z, t\right)=$ $e A_{0,1} / m c^{2}$. The subscripts 0 and 1 distinguish the right-moving (guided) pulse and the left-moving (guiding) pulses, respectively. The phases of the waves are $\theta_{0}=\left(k_{0} z-\omega_{0} t\right)$ and $\theta_{1}=\left(k_{1} z+\omega_{1} t\right)$, and we choose $|\Delta \omega|=\left|\omega_{0}-\omega_{1}\right| \ll \omega_{0}$. It is further assumed that the pulses are propagating through a tenuous plasma $\omega_{p} \ll \omega_{0,1}$, so that the phase and group velocities of both pulses are close to the speed of light $c$, and, to lowest order in $\omega_{p} / \omega_{0}, k_{0} \approx k_{1} \approx \omega_{0} / c$.

When both lasers are of nonrelativistic intensity, $a_{0,1} \ll 1$, the only nonlinear coupling mechanism between them is through the ponderomotively generated density perturbation $\delta n / n_{0}=\hat{n} \exp i\left(\theta_{0}+\theta_{1}\right)+$ c. c. This perturbation is generated by the $\vec{v}_{1} \times \vec{B}_{0}+\vec{v}_{0} \times \vec{B}_{1}$ force, where $\vec{v}_{0,1}=c \vec{a}_{0,1}$. Serving as an index grating with the wavenumber $k=k_{0}+k_{1}$, oscillating at the difference frequency $\Delta \omega$, this density perturbation scatters the left-going pulse $a_{1}$ into the right-going pulse $a_{0}$, and vice versa. This is precisely the mechanism responsible for the stimulated Raman backscattering in the plasma, which received a lot of theoretical $[17,18]$ and experimental $[19,20]$ attention. Below we demonstrate how this interaction between the counter-propagating lasers leads to the nonlinear focusing of one (or both) pulses.

The nonlinear interaction between the $a_{0}$ and $a_{1}$ is calculated by substituting the modified plasma density into the paraxial wave equations for the right and left-moving pulses. For example, for the guided pulse obtain $2 i k_{0}\left(\frac{\partial}{\partial z}+\frac{1}{c} \frac{\partial}{\partial t}\right) a_{0}+\nabla_{\perp}^{2} a_{0}=-k_{p}^{2} \chi_{0} a_{0}$, where $\chi_{0}$ is the nonlinear index of refraction witnessed by the guided beam, given by

$$
\chi_{0}(\tau)=\frac{2 \omega_{0}^{2}\left|a_{1}\right|^{2}}{\omega_{p}} \int_{0}^{\infty} d u \sin \left(\omega_{p} u\right) e^{i \Delta \omega u} \frac{a_{0}(\tau-u)}{a_{0}(\tau)}
$$

where $\tau=t-z / c$ is a co-moving with the guided pulse coordinate, and the guiding pulse is assumed very long. 
The advantage of using counter-propagating pulses is made apparent by Eq. (1): the longitudinal gradient scale of laser intensity becomes very small (about $\lambda_{0} / 2$ ), greatly reducing the laser power required to produce a given density modulation. As Eq. (1) indicates, the EIG of a given longitudinal laser slice $\tau$ is determined by the laser intensity at all earlier instances $\tau^{\prime}<\tau$, so that the degree of focusing experienced by a short pulse is not uniform along the pulse and determined by its longitudinal profile. One finds that, if the pulse intensity drops off faster than exponentially in $|\tau|$, the ratio $a_{0}(\tau-u) / a_{0}(\tau)$ decreases with $|\tau|$. For example, for a longitudinally Gaussian profile $a_{0}=\alpha_{0} \exp \left(-\tau^{2} / 2 \tau_{L}^{2}\right)$ the leading edge $\tau<-\tau_{L}$ slowly erodes because $\chi_{0}(\tau) \propto \tau_{L}^{2} / \tau^{2}$. However, for $a_{0}=\alpha_{0} \sec \left(\tau / \tau_{L}\right)$ (exponential decay in $|\tau|)$ the leading edge is uniformly focused: $\chi_{0}=2\left|a_{1}\right|^{2} \omega_{0}^{2} \tau_{L}^{2} /\left(1+\omega_{p}^{2} \tau_{L}^{2}\right)$ for $\Delta \omega=0$. Since the plasma does not respond on a time scale faster than $1 / \omega_{p}$, the guiding is reduced for ultra-short pulses.

For a guided pulse of duration $\tau_{L} \gg \min \left(1 / \Delta \omega, 1 / \omega_{p}\right)$ the nonlinear index of refraction is independent of $\tau$, ensuring uniform focusing of the entire guided pulse: $\chi_{0}=2\left|a_{1}\right|^{2} \omega_{0}^{2} /\left(\omega_{p}^{2}-\right.$ $\left.\Delta \omega^{2}\right)$. Thus, the nonlinear index of refraction acquires a transverse variation proportional to the transverse profile of the guiding laser. Two long transversely Gaussian beams focus each other if $\Delta \omega<\omega_{p}$. Since this type of nonlinear guiding relies on a rather delicate mechanism of generating an index grating with a very short wavelength, and then backscattering off this grating, we use direct Particle in Cell (PIC) simulation in a slab geometry to demonstrate the existence of the mutual focusing effect. Then we develop a simplified analytical description of the nonlinear guiding of Gaussian pulses in the slab and cylindrical geometries, interpret the simulation results, and address problem (ii).

To simulate the mutual focusing of two identical laser pulses we use a $2 \mathrm{D}$ version of the relativistic electromagnetic PIC code VLPL (Virtual Laser Plasma Lab) [21] running on a single processor workstation. The grid size was $4000 \times 120$ with $4 \times 10^{6}$ electrons on it. The two counter-propagating laser pulses with wavelengths $\lambda_{1}=\lambda_{0}=1 \mu \mathrm{m}$ are focused to the spotsizes $\sigma_{0}=\sigma_{1}=3 \mu \mathrm{m}$ at their corresponding entrances into a $400 \mu \mathrm{m} \times 40 \mu$ slab of $n_{0}=10^{19} \mathrm{~cm}^{-3}$ plasma. The normalized vector potentials of both lasers at their respective 
foci are equal to $\alpha_{0}=0.07$. The intensity gray scale plots for the right-moving pulse in vacuo and in the plasma are shown in Figs. 1(b) and 1(c), respectively. The snapshots at $t=500 \lambda_{0} / c$ of the on-axis $(x=0)$ laser intensities for both cases are plotted versus the propagation distance in Fig. 1(a).

As Fig. 1(a) demonstrates, in the presence of the plasma the intensity of the right-going laser $\left|a_{0}\right|^{2}\left(z=L_{g}\right)$ is increased by a factor 2. Thus, the nonlinear interaction between the lasers strongly reduces laser spreading, confirming the electromagnetically induced guiding. The simulation was run till $t=800 \lambda_{0} / c$, with no degradation of the mutual focusing. The driven density modulation remains coherent despite the modest plasma heating, which raises the temperature to about $500 \mathrm{eV}$. Note that for the parameters of the simulation the growth rate of the Raman backscattering (RBS) instability of the guiding beam $\gamma_{R B S} \ll \omega_{p}$, so that the frequency of the guided beam is outside of the RBS amplification bandwidth. RBS can grow from plasma noise, causing the beam reflection from the plasma. However, the saturated reflectivity is quite small for the underdense plasma assumed in the simulation [18].

A simplified analytical treatment of electromagnetically induced guiding can be developed by assuming that the guided laser pulse has a Gaussian transverse profile when it enters the plasma, and that it remains such in the plasma. Flat and cylindrical beams are considered separately because of the different laser intensity normalizations. The case of flat beams is important because it provides the basis for comparison with the results of the 2D PIC simulation. Guided beam is assumed to have an intensity profile given by $|a|_{0}^{2}=\left(1 / R_{0}\right) \alpha_{0}^{2} \exp \left[-x^{2} / 2 R_{0}^{2} \sigma^{2}\right]$ in slab geometry and $|a|_{0}^{2}=\left(1 / R_{0}^{2}\right) \alpha_{0}^{2} \exp \left[-r^{2} / R_{0}^{2} \sigma^{2}\right]$ in cylindrical geometry, where $R_{0}$ is the dimensionless spotsize. Applying the source-dependent expansion to the envelope equations for $a_{0}$ similarly to the way it was done in Ref. [8], the equation for the normalized radius of a guided beam is derived:

$$
\frac{d^{2} R_{0}}{d \bar{z}^{2}}=\frac{1}{R_{0}^{3}}+\frac{2 k_{p}^{2} \sigma^{2}}{\sqrt{2 \pi} R_{0}^{2}} \int_{-\infty}^{+\infty} d y y e^{-y^{2} / 2 R_{0}^{2}} \frac{\partial \chi_{0}}{\partial y}
$$

for flat beams, and 


$$
\frac{d^{2} R_{0}}{d \bar{z}^{2}}=\frac{4}{R_{0}^{3}}+\frac{4 k_{p}^{2} \sigma^{2}}{R_{0}^{3}} \int_{0}^{+\infty} d \rho \rho^{2} e^{-\rho^{2} / R_{0}^{2}} \frac{\partial \chi_{0}}{\partial \rho}
$$

for round beams, where $y=x / \sigma$ and $\rho=r / \sigma$ are the normalized transverse coordinates, and $d / d \bar{z}=2 k_{0} \sigma^{2}(\partial / \partial z+\partial / c \partial t)$.

To interpret the results of the PIC simulation, assume that the guiding pulse is also Gaussian with radius $R_{1}$, simplifying Eq. (2) to yield

$$
\frac{d^{2} R_{0}}{d \bar{z}^{2}}=\frac{1}{R_{0}^{3}}-\frac{4 k_{p}^{2} \sigma^{2} \alpha_{0}^{2} \omega_{0}^{2}}{\omega_{p}^{2}-\Delta \omega^{2}} \frac{R_{0}}{\left(R_{0}^{2}+R_{1}^{2}\right)^{3 / 2}},
$$

confirming that the lasers focus each other if $\Delta \omega<\omega_{p}$. According to Eq. (4), the two lasers can, in principle, form a mutually guided state $R_{0}=R_{1}=1$ if $\alpha_{0} \approx 0.05$. This state is, however, unstable, so that it is never reached in a time-dependent situation, such as the one modeled by the PIC simulation. At the beginning of the plasma region the guided pulse interacts with the diffracted guiding pulse of much lower intensity. This explains why a somewhat higher $\alpha_{0}=0.07$ was needed in the simulation to observe the EIG.

We now address the second problem: focusing of an ultra-short tightly focused laser pulse by a counter-propagating lower intensity long laser pulse. One application of such ultra-short pulses is laser-wakefield acceleration. To increase the final energy of accelerated electrons, two quantities have to be maximized: the amplitude of the plasma wave left behind the pulse and the total acceleration length. Large plasma wake is excited by a laser pulse of a duration shorter than the plasma period. Assuming that the guided pulse has a longitudinal profile $a_{0}=\alpha_{0} \sec \left(\tau / \tau_{L}\right)$, it can be demonstrated that the optimal pulse duration for wake excitation is $\tau_{L}=1.2 / \omega_{p}$. The guiding pulse should propagate without diffraction over the entire region of the plasma since its propagation is unaffected by the short guided pulse.

It has been known for some time [28] that apertured Bessel beams with sharply peaked radial profiles propagate without diffraction over the distances much exceeding the Rayleigh length. Such beams transport energy within a narrow spot $\sigma_{1} \ll W$ over the distance of order $L_{g}=2 \pi W \sigma_{1} / \lambda_{1}$, where the laser intensity profile is given by $\left|a_{1}\right|^{2}=\alpha_{1}^{2} J_{p}^{2}\left(r / \sigma_{1}\right)$, and the beam is apertured at the radius $W \gg \sigma_{1}[28]$. The total power of a Bessel beam 
is given by $P_{B p} / P_{0}=\pi W \sigma_{1}\left|\alpha_{1}\right|^{2} / \lambda_{0}^{2}$, where $P_{0}=m c^{3} / r_{e}=8.0 \mathrm{GW}$ and $r_{e}=e^{2} / m c^{2}$ is the classical electron radius. Therefore, the propagation distance is proportional to the beam power. Below we demonstrate how low-intensity Bessel beams can be used to guide ultra-intense short Gaussian beams. Apart from the basic interest to nonlinear laser-plasma science, an experimental implementation of this novel guiding scheme would enable, for example, detailed studies of the stability of guided laser propagation in over-moded channels [22]. Note that directly generating a plasma wake with a Bessel beam offers no advantages over a Gaussian beam since the product of the peak on-axis intensity and the propagation distance is, approximately, the same for the equal power Gaussian and Bessel beams [23]. Guiding can also be achieved by generating a plasma density depression on axis through the hydrodynamic expansion of laser-produced plasmas [24] or in a capillary discharge [25].

The choice of $p$, the order of the Bessel beam, depends on the frequency detuning of the lasers. The nonlinear index of refraction $\chi_{0}$ changes sign when $\Delta \omega$ crosses $\omega_{p}$. If $\Delta \omega<\omega_{p}, 1 / \tau_{L}$, the zeroth-order Bessel beam generates the $\chi_{0}$ with a maximum on axis (as required for guiding). For example, for $\Delta \omega=0$ the leading edge of the pulse experiences $\chi_{0}=2\left|a_{1}\right|^{2} \omega_{0}^{2} \tau_{L}^{2} /\left(1+\omega_{p}^{2} \tau_{L}^{2}\right)$. In the opposite regime of $\Delta \omega>\omega_{p}, 1 / \tau_{L}$ the index of refraction is negative: $\chi_{0}=2\left|a_{1}\right|^{2} \omega_{0}^{2} /\left(\omega_{p}^{2}-\Delta \omega^{2}\right)$. Therefore, to ensure focusing, the appropriate guiding pulse is the first-order Bessel beam, which has an intensity minimum on axis. First we consider guiding by the zeroth-order Bessel beam.

Numerical modeling of guiding a short pulse by the Bessel beam requires a 3D PIC simulation and imposes very strict limitations on the spatial and temporal resolution and the size of the simulation domain. This makes direct PIC simulations very difficult, and we rely on our analytical results. The required for guiding power of the backward-moving Bessel beam $P_{B 0}$ is estimated by substituting $\left|a_{1}\right|^{2}=\alpha_{1}^{2} J_{0}^{2}\left(r / \sigma_{1}\right)$ into Eq. (3) and assuming $R_{0}=1:$

$$
k_{0}^{2} \sigma^{2}\left|\alpha_{1}\right|^{2} \frac{2 \omega_{p}^{2} \tau_{L}^{2}}{1+\omega_{p}^{2} \tau_{L}^{2}}\left[t \frac{d}{d t}\left(e^{-t} I_{0}(t)\right)\right]=-1
$$

where $t=\sigma^{2} / 2 \sigma_{1}^{2}$ and $I_{0}(t)$ is a modified Bessel function. The strongest focusing occurs for 
$\sigma_{1} \approx 0.8 \sigma$. Since the guiding distance $L_{g}$ is related to the beam power $P_{B 0}$, Eq. (5) can be rewritten as a power threshold for the EIG:

$$
P_{B 0}=1.27 \mathrm{GW} \frac{1+\omega_{p}^{2} \tau_{L}^{2}}{\omega_{p}^{2} \tau_{L}^{2}} \frac{L_{g}}{k_{0} \sigma^{2}} .
$$

The EIG threshold $P_{B 0}$ differs from the relativistic guiding threshold $P_{c 1}$ in two respects: first, it is independent of the plasma density, enabling electromagnetically-induced channeling in very tenuous plasmas, and, second, it depends on the propagation distance. The qualitative reason for the first difference is that, although smaller plasma density reduces the effectiveness of the nonlinear guiding for the same fractional density perturbation $\hat{n}$, a smaller laser intensity is needed to generate the same $\hat{n}$, according to Eq. (1). The theory of the EIG, developed in this paper, assumes a linear plasma response, which is only valid when $\hat{n}<1$. Combining this restriction with the guiding condition, given by Eq. (5), imposes an upper limit on the intensity of the guided pulse: $\alpha_{0} \leq \lambda_{0} \sigma / \lambda_{p}^{2}$. More extensive numerical study is needed to fully understand how the guiding is affected when the linear theory breaks down, but it is reasonable to expect that the guiding saturates and weakens at higher intensities of the guided pulse.

As evident from Eq. (3), the focusing strength is determined by the curvature of the $\chi_{0}$ rather than by its absolute value. Therefore, a possible solution to the nonlinear saturation of the EIG is to use a first-order Bessel beam, which has an intensity node on axis. The density perturbation on axis vanishes while its curvature does not, providing the guiding for the counter-propagating short pulse. Moreover, using the $J_{1}$ beam detuned by $\Delta \omega>$ $\omega_{p}, 1 / \tau_{L}$ enables guiding ultra-short pulses of almost arbitrary longitudinal shapes (including Gaussian) without the leading edge erosion. The guiding condition similar to Eq. (5) can be derived for the $J_{1}\left(r / \sigma_{1}\right)$ beam. The strongest focusing occurs for $\sigma_{1}=1.04 \sigma$, yielding $k_{0}^{2} \sigma^{2} \alpha_{1}^{2}=5\left[\left(\Delta \omega / \omega_{p}\right)^{2}-1\right]$, from which the power threshold condition can be derived:

$$
P_{B 1}=3.6 \mathrm{GW}\left(\Delta \omega^{2} / \omega_{p}^{2}-1\right) \frac{L_{g}}{k_{0} \sigma^{2}} .
$$

The linear theory breaks down at $r \approx \sigma$ if $\alpha_{0}>2.7 \lambda_{0} \sigma / \lambda_{p}^{2}$. In practice, this limitation is likely to be over-stringent since the nonlinear saturation of the EIG in a limited spatial region 
may not strongly influence the overall focusing. As a numerical example, consider guiding a $\tau_{L}=10 \mathrm{fs}$ long, $\sigma=18 \mu \mathrm{m}$ wide laser pulse through $1 \mathrm{~cm}$ of $n_{0}=10^{19} \mathrm{~cm}^{-3}$ plasma. For $\Delta \omega / \omega_{p}=1.5$ the threshold power of the Bessel beam $P_{B 1} \approx 16 \mathrm{GW}$. This beam can guide a pulse with a normalized vector potential up to at least $\alpha_{0}=0.5$, or $2.2 \mathrm{TW}$. Although an appreciable long-wavelength $\left(\lambda=\lambda_{p}\right)$ plasma wake is generated by the guided pulse, it will not interfere with the short-wavelength density perturbation as long as the plasma behaves linearly $\left(\alpha_{0}<2.7 \lambda_{0} \sigma / \lambda_{p}^{2}<1\right)$, and the total density perturbation is a linear superposition of the short- and long-wavelength wakes.

In conclusion, we demonstrated analytically and numerically the electromagneticallyinduced guiding of two counter-propagating lasers in the plasma at intensities much below the threshold for relativistic guiding. In addition, we described the technique for electromagnetic channeling of an ultra-short laser pulse by a lower-intensity counter-propagating Bessel beam.

The authors gratefully acknowledges helpful discussions with J. S. Wurtele. This work was supported by the United States Department of Energy (US DoE) contract No. DEAC02-CHO-3073. 


\section{REFERENCES}

[1] M. D. Perry and D. Mourou, Science 264, 917-924 (1994); D. Mourou and D. Umstadter, Phys. Fluids B 4, 2315 (1992).

[2] C. P. J. Barty et. al., Opt. Lett., 21, 668 (1996).

[3] A. K. Lal et. al., Phys. Rev. Lett. 78, 670 (1997).

[4] S. E. Harris, Phys. Rev. Lett. 77, 5357 (1996).

[5] D. Umstadter et. al., Science, 273, 472 (1996).

[6] T. Tajima and J. M. Dawson, Phys. Rev. Lett. 43, 267 (1979); E. Esarey et. al., IEEE Trans. Plasma Science 24, 252 (1996), and references therein.

[7] C. E. Max et. al., Phys. Rev. Lett. 33, 209 (1974); A. B. Borisov, Phys. Rev. A 45, 5830 (1992); X. L. Chen and R. N. Sudan, Phys. Rev. Lett. 70, 2082 (1993); A. Pukhov and J. Meyer-ter-Vehn, Phys. Rev. Lett. 76, 3975 (1996).

[8] E. Esarey et. al., Phys. Rev. Lett. 72, 2887 (1993).

[9] P. Monot et. al., Phys. Rev. Lett. 74, 2953 (1995); M. Borghesi et. al., Phys. Rev. Lett. 78, 879 (1997).

[10] P. Sprangle et. al., Phys. Rev. Lett. 64, 2011 (1990).

[11] T. M. Antonsen, Jr., and P. Mora, Phys. Rev. Lett. 69, 2204 (1992); T. M. Antonsen, Jr., and P. Mora, Phys. Fluids B 5, 1440 (1993).

[12] J. Krall et. al., Phys. Rev. E. 48, 2157 (1993).

[13] N. E. Andreev et. al., Phys. Plasmas 2, 2573 (1995).

[14] K.-J. Boller et. al., Phys. Rev. Lett. 66, 2593 (1991); S. E. Harris, Physics Today 50, 36 (July 1997).

[15] S. E. Harris, Opt. Lett. 192018 (1994). 
[16] P. Gibbon and A. R. Bell, Phys. Rev. Lett. 61, 1599 (1988); P. Gibbon, Phys. Fluids B 2, 2196 (1990).

[17] C. J. McKinstrie, A. Simon, Phys. Fluids, 29, 1959 (1985); T. Kolber et. al., Phys. Plasmas 2, 256 (1996); N. E. Andreev et. al., Phys. Plasmas 2, 2573 (1995).

[18] G. Shvets et. al., Phys. Plasmas, 4, 1872 (1997).

[19] C. Rousseaux et. al., Phys. Rev. Lett. 74, 4655 (1995); V. Malka et. al., Phys. Plasmas 3, $1682(1996)$.

[20] M. J. Everett et. al., Phys. Rev. Lett. 74, 1355 (1995).

[21] A. Pukhov and J. Meyer-ter-Vehn, APS Bulletin 41, 1502 (1996).

[22] G. Shvets and J. S. Wurtele, Phys. Rev. Lett. 73, 3540 (1994).

[23] B. Hafizi, E. Esarey, and P. Sprangle, Phys. Rev. E 55, 3539 (1997).

[24] C. G. Durfee and H. M. Milchberg, Phys. Rev. Lett. 71, 2409 (1993); C. G. Durfee et. al., Phys. Rev. E 51, 2368 (1995); H. M. Milchberg et. al., Phys. Plasmas 3, 2149 (1996).

[25] A. Zigler et. al., Journ. Opt. Soc. of America B 13, 68 (1996).

[26] A. Yariv, Optical Electronics, Chapter 2, Saunders College Publishing, Philadelphia, 1991.

[27] C. G. Durfee et. al., Opt. Lett. 19, 1937 (1994); T. M. Antonsen, Jr. and P. Mora, Phys. Rev. Lett. 744, 4440 (1995).

[28] J. Durnin et. al., Phys. Rev. Lett. 58, 1499 (1987).

[29] C. E. Clayton et. al., Phys. Rev. Lett. 70, 37 (1993). 


\section{FIGURES}

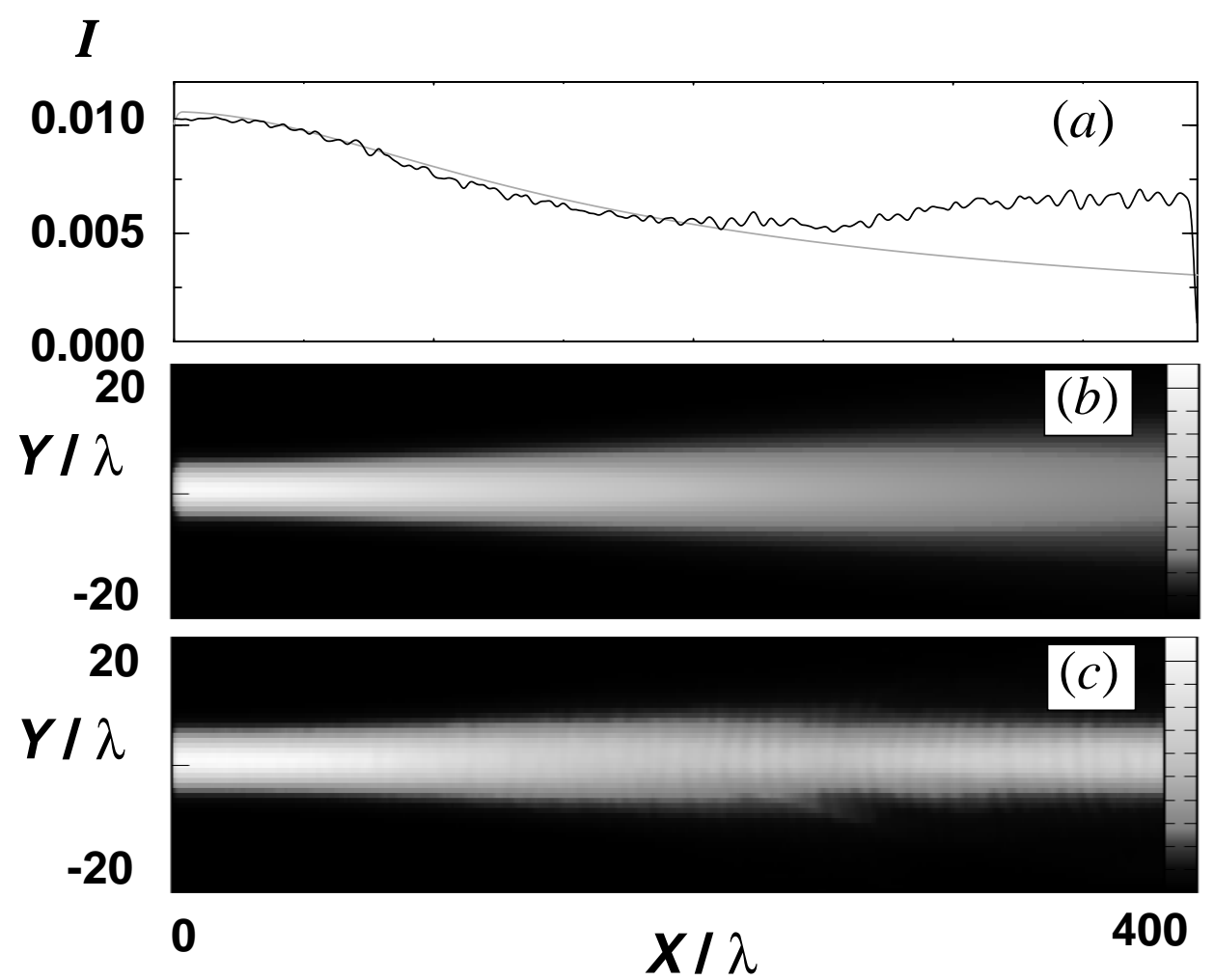

FIG. 1. (a) On-axis intensity of a right-going laser focused at $z=0$ in vacuum (dashed line) and in the plasma (solid line). Contour plot of the intensity of the right-going laser in vacuum (b) and in the plasma (c). 